\title{
XELIRINOX Regimen a Triple Combination of XELoda, IRINotecan and OXaliplatin in First-line Treatment of Metastatic Pancreatic Adenocarcinoma
}

\author{
Haider Y. Shukur \\ Najaf Cancer Clinic (NCC), Department of Oncology, Jabir ibn Hayyan Medical University, Iraq
}

Received July 18, 2019; Revised September 5, 2019; Accepted September 20, 2019

Copyright $\mathrm{O} 2020$ by authors, all rights reserved. Authors agree that this article remains permanently open access under the terms of the Creative Commons Attribution License 4.0 International License

\begin{abstract}
FOLFIRINOX CT has shown notable responses in patients with metastatic PAC and has significantly improved prognosis. However, FOLFIRINOX is currently not frequently applied to all patients because of its high incidence of adverse events. Alternated XELIRINOX may be better for its acceptance in more patients' population. In this study, we evaluated the efficacy and safety of XELIRINOX in metastatic PAC. A total of 40 metastatic PAC patients were treated with XELIRINOX (Capcitabine substitute 5-FU, 75\% Irinotecan dose and 100\% Oxaliplatin dose) between January 2014 and January 2018 in our NCC. All 40 patients were evaluated with CR occur only in $2.5 \%$ (1/40) with an OAR of $35 \%(14 / 40)$. The frequent grade $3 / 4$ adverse events are neutropenia (30\%) and diarrhea (30\%). No treatment-related death was observed. The median OS and median PFS is 10.5 months and 7.5 months, respectively. In conclusion, XELIRINOX had significantly improved tolerance with near similar efficacy to FOLFIRINOX. These findings may provide evidence for the use of XELIRINOX in more patient population with metastatic pancreatic adenocarcinoma.
\end{abstract}

Keywords XELIRINOX, CT, Pancreatic Cancer, Capecitabine, Irinotecan, Oxaliplatin, Triplet Regimen

\section{Introduction}

During the year 2018 in the United State, an estimated 55,440 people will be diagnosed with exocrine PC, where the majority of these tumors $(85 \%)$ are adenocarcinomas arising from the ductal epithelium, and approximately 43,330 people will be dying of the disease as demonstrated by Islami et al.[1] Furthermore, the 5-year relative survival rate was only $8 \%$, and that of the distant stage was only $3 \%$ as stated by Siegel et al.[2]

Siegel et al., because of its aggressive character and the fact that most patients present with relatively advanced disease, most die from the disease, So it considers the fourth most common cause of cancer -related death among U.S. men (after lung, prostate \&colorectal cancer) and women (after lung, breast \& colorectal cancer).[1]

Simard et al. notice that, although incidence is roughly equal in both sexes, an African American has a higher incidence of pancreatic cancer than white Americans. [3]

Furthermore, the incidence of PC in the US increased from 1999 to 2008, possibly because of the increasing prevalence of obesity, an aging population \& other unknown factors as notified by Eheman et al. [4]. But Worni et al. saw mortality rates have remained largely unchanged. [5]

Fogel et al. stated that, the surgical resection offers the only chance of cure. However, only 15 to $20 \%$ of patients have resectable disease at initial diagnosis; the majority has either locally advanced or metastatic cancer as mentioned by Warsame et al. $[6,7]$

Other than surgical resection, systemic CT is the only major treatment that can improve survival in patients with locally advanced or metastatic PC. Twenty years ago, gemcitabine (GEM) replaced an IV bolus 5-fluorouracil (5-FU) as the main chemotherapeutic drug for treating advanced PC because a modest survival increase (5.65 versus 4.41 months) and more clinical benefits were found in a Phase III clinical trial as confirmed by Burris et al. [8]

Di Marco et al. said that, although the GEM monotherapy had been the gold standard for pancreatic cancer, but the response rate of GEM monotherapy remains low (approximately 10\%), and an improved strategy is desperately needed [9].

Therefore, numerous clinical trials combined GEM with other anti-tumour agents to increase the anti-tumour effects, but most such studies were unable to demonstrate the 
superiority of or a significant improvement in OS for GEM combination therapy; only GEM combined with capecitabine and erlotinib have shown promise as confirmed by Warsame et al. [7]

Recently, Conroy et al. in the PRODIGE 4/ACCORD 11 randomized trials, a four-drug regimen called FOLFIRINOX, consisting of folinic acid, 5-fluorouracil, irinotecan and oxaliplatin, was demonstrated to prolong OS compared to GEM monotherapy (11.1 months versus 6.8 months). These results suggested that this combined regimen should be used in clinical practice as a first-line option for advanced PC patients. [10]

Shortly thereafter, Von Hoff et al. explored thata regimen of GEM and albumin-bound paclitaxel was shown to have statistically significant survival benefits in OS and PFS, thus providing another choice for treating advanced PC. [11]

However, FOLFIRINOX appears to be more effective than GEM/NAB-P as documented by Chan et al. [12]

Although FOLFIRINOX is a first-line option for patients with metastatic PAC, there is a controversy about whether the survival benefits of the four-drug combination regimen outweigh the associated toxicities as stated by Gresham et al. [13]

However, the survival gain comes at a cost. Compared to GEM , FOLFIRINOX is reported with a higher rate of grade $3 / 4$ adverse events, including neutropenia $(45.7 \%$ versus. $21 \%)$, fatigue $(23.6 \%$ versus. $17.8 \%)$, vomiting $(14.5 \%$ versus. $8.3 \%)$, diarrhea ( $12.7 \%$ versus. $1.8 \%)$, and sensory neuropathy $(9 \%$ versus. $0 \%)$. For this reason, FOLFIRINOX was only used in patients $\leq 76$ years old who have a good performance status (ECOG 0 or 1 ) at full dose, and the tolerability still remains a main concern as revealedbyConroy et al. $[10,14]$.

As a result, an increasing number of studies have aimed to improve chemotherapeutic or targeted therapy regimens to reduce toxicity without compromising efficacy. Using dose attenuation, Mahaseth et al. reported a significantly reduced incidence of adverse events with a similar median OS (9.0 versus. 11.1 months compared to full dose) [15]. Similarly, Stein et al. demonstrated that reduction of irinotecan and bolus 5 -fluorouracil by $25 \%$ resulted in significantly decreased neutropenia, vomiting, and fatigue, with a relatively high response rate of $35.1 \%$ [16].

To decrease the side effects of FOLFIRINOX and increase its tolerability, we planned this study to assess the effectiveness and toxicities of this alternated XELIRINOX regimen in patients with metastatic PAC. We hope more patients can benefit from this alternated XELIRINOX regimen.

Capecitabine is an oral fluoropyrimidine carbamate prodrug designed to generate 5-FU preferentially in tumor cells due to the high concentration level of thymidine phosphorylase enzyme. This allows to mimic continuous 5-FU infusion at the tumor site and to reduce exposure of adjacent healthy tissues without causing discomfort and complications related to IV administration. It has been widely used for the treatment of colorectal cancers and breast cancer as noticed by Mazard et al. [17]

Cassidy et al. there are different phase III trials have shown that capecitabine is at least as active and effective as 5-FU in the first-line treatment of $\mathrm{mCRC}$, with a superior safety profile. [18]

Moreover, Machen et al the use of capecitabine instead of 5-FU, either with irinotecan or oxaliplatin, confirmed the activity and efficacy of the drug. [19]

Mazard et al .there isa phase I trial showed promising results of the combination of capecitabine, oxaliplatin and irinotecan in mCRC subjects and suggested that this tri-therapy may provide valuable therapeutic alternative, especially in patients with GI cancer. [17]

Based on these results, the triple combination of capecitabine with oxaliplatin and irinotecan appears to be an interesting regimen. Therefore, it is of interest to explore the possibility to replace IV 5-FU and leucovorin in the FOLFIRINOX regimen with capecitabine and to assess the efficacy and tolerability of this modified regimen in the treatment of metastatic PAC, that could simplify the treatment delivery and reduce the complications related to the central venous catheter compared to infusional $5-\mathrm{FU}$, as used in the FOLFIRNOX regimen.

Machen et al. stated that there are different schedules of capecitabine emerged from phase I trials. [20] It is worth noting that mathematical methods applied to the definition of the ideal treatment schedule suggested that the optimal duration of treatment with capecitabine is 7 days and predicted that drug delivery beyond 7 days could contribute to toxicity, with diminishing anticancer benefit as noticed by Traina et al. [21]

Moreover, a randomised phase II trial conducted by Scheithauer et al. demonstrated that a dose intensified bimonthly combination of oxaliplatin plus capecitabine administered for 7 days followed by 7 days rest is as safe and feasible as the combination of oxaliplatin on day 1 with capecitabine administered from day 1 to day 14 every 3 weeks, with higher RR and PFS for the bimonthly regimen. [22]

\section{Patients and Methods}

\subsection{Patient Eligibility}

This was a prospective study involving metastatic PAC receiving XELIRINOX from January 2014 to January 2018 at the Najaf Cancer Clinic (NCC), Affiliated clinic, Jaber Ibn Hayyan Medical University. Patients, who were histologically diagnosed with metastatic PAC and were prescribed XELIRINOX as first line treatment, were eligible for inclusion. In all cases, metastatic PC was diagnosed by a multidisciplinary team, according to the National Comprehensive Cancer Network definitions. 


\subsection{Inclusion Criteria}

1) WHO-PS of0-2

2) Age 40 to 75 years

3) GFR $\geq 30 \mathrm{ml} / \mathrm{min}$

4) Patient with solitary kidney was included

5) Written informed consent had to be given.

\subsection{Exclusion Criteria}

1) WHO-PS $\geq 3$

2) Abnormal biochemistry (i.e., bilirubin 1.3-ULN, alkaline phosphatase 5-ULN, AST/ALT 5-ULN).

3) Inadequate bone marrow, liver or renal functions;

4) Double malignancies

5) Older than 75 years.

\subsection{Pretreatment Evaluation Included}

All patients were evaluated every four cycles of XELIRINOX (every two months) using high-resolution computed tomography (HRCT) scan chest- abdomen and enhanced MRI of the liver. The serum tumor markers CA19-9 \& CEA levels were examined pretreatment as baseline and at each time of tumor response assessment.

Physical examination and laboratory tests $(\mathrm{CBC}$, and chemistry) were performed every time before $\mathrm{CT}$. Tumor response was categorized in $\mathrm{CR}, \mathrm{PR}, \mathrm{SD}$, and $\mathrm{PD}$ according to the Response Evaluation Criteria in Solid Tumors (version 1.1) mentioned by Eisenhauer et al. [23]

Adverse events were graded according to the National Cancer Institute Common Terminology Criteria for Adverse Events (version 4.0). The OS was defined as the duration from the beginning of CT till the date of death of any cause. PFS was defined as the duration from the beginning of CT till the date of disease progression or death. Patients without event were censored at the last follow-up date (February 1st, 2018). For patients with XELIRINOX, information was collected at the moment of admission. While patients who had discharged were followed up every 3 months in a clinic by physical examination, laboratory tests, chest-abdomen HRCT, enhanced MRI of the liver \& markers.

\subsection{Treatment Schedule}

The XELIRINOX regimen consisted of irinotecan 135 $\mathrm{mg} / \mathrm{m}^{2}$ i.v. in $250 \mathrm{ml}$ of $\mathrm{NaCl}, 0.9 \%$ over $1 \mathrm{~h}$, followed immediately by oxaliplatin $85 \mathrm{mg} / \mathrm{m}^{2}$ i.v. in $250 \mathrm{ml}$ dextrose $5 \%$. Capecitabine was administered at the dose of $2000 \mathrm{mg} / \mathrm{m}^{2}$ per day orally in two divided doses from day 1 to day 7 . Treatment was repeated every 2 weeks and administered until evidence of disease progression, unacceptable toxicity, patient refusal or for a maximum of 12 cycles.

For all patients, this treatment was preceded by premedication as antiemetic (dexamethasone $16 \mathrm{mg}$ and
5-HT3 receptor antagonists $8 \mathrm{mg}$ ondansetron), Ranitidine $150 \mathrm{mg}$ and diphenhydramine maleate ampoule all are administered intravenously in $100 \mathrm{ccs}$ normal saline solution over $15 \mathrm{~min}$. just before chemotherapy, also vitamin B6 ampoule $50 \mathrm{mg} / \mathrm{ml}$ given to reduce neurotoxicity induced by oxaliplatin. Furthermore B6 tablets $50 \mathrm{mg}$ BID \& celecoxib $200 \mathrm{mg}$ capsule once daily during capcitabine uses to reduce incidence of hand foot syndrome \& peripheral neuropathy. Atropine $0.25 \mathrm{mg}$ subcutaneously was given to treat the cholinergic syndrome, and repeated as prophylaxis of future events in the following cycles.All these premedication were used to enhance the tolerance against XELIRINOX-related adverse events. All patients in the cohort were offered alternated XELIRINOX (2-weekly schedule) and evaluated every 4 cycles.

CBS and other necessary blood tests were regularly performed every two weeks prior to each cycle. Biliary stenting and drainage procedures were performed in patients with jaundice and tapping ascetic fluid were performed if presentprior to starting treatment.

Toxicities were graded according to the NCI CTC version 4.0. Treatment was delayed until recovery in case of neutrophils $<1.000$ per $\mathrm{mm}^{3}$, platelets $<100.000$ per $\mathrm{mm}^{3}$ or diarrhoea or stomatitis grade $>1$ on the planned day of treatment. In the case of peripheral neurotoxicity grade $>2$, oxaliplatin was interrupted.

In the case of previous dose-limiting toxic effects, treatment delayed was permitted until recovery, then continued after resolution of the event with same doses of oxaliplatin, irinotecan and capecitabine dose reduced by $25 \%$, except in the case of grade 3-4 diarrhoea, when only irinotecan and capecitabine doses were reduced by $25 \%$. Loperamide $2 \mathrm{mg}$, orally every $2 \mathrm{~h}$, and oral rehydration were prescribed in case of delayed diarrhoea. In the case of life-threatening toxic effects, treatment was definitively interrupted or continued at doses reduced by $50 \%$. Prophylactic treatment with G-CSF for neutropenia was recommended.

Patients discontinued the study in the event of unacceptable toxic effects or evidence of progressive disease, or at their request. For those patients with CR, PR or SD, the duration of XELIRINOX will be as long as 12 cycles and then keep patient on close follow up. When disease, recurrent, XELIRINOXis still the first choice for patients with a good performance score.

\subsection{Treatment Assessments}

During treatment, the assessment, including toxicity and response evaluation:

At the end of fourth cycles, patients were assessed with physical examination, blood chemistry, blood count and creatinine were performed every 2 weeks. Evaluation of tumour response was performed with a CT scan of the chest-abdomen every 8 weeks according to the standard RECIST criteria .The best OAR for each patient was 
reported as mentioned by Eisenhauer et al.[23]

Then, responding patients complete further four cycles then re-evaluate completely and planned to received last four cycles then re-evaluate completely and keep on follow up every 3 months. In other hand, patients with disease progression after fourth cycles shift to BSC.

\section{Statistical Analysis}

Data were analyzed using SPSS software (version 22). Numerical data were expressed as median and range as appropriate. Qualitative data were expressed as a percentage. The survival curves were estimated using the Kaplan-Meier technique.

\section{Results}

Table 1. Patient Clinicopathological Characteristics

\begin{tabular}{|l|l|}
\hline \multicolumn{1}{|c|}{ Characteristic } & \multicolumn{1}{|c|}{$\begin{array}{c}\text { No. of patients } \\
\text { N=40 }\end{array}$} \\
\hline Age -Mean(Range ) & $61.3(40-75)$ \\
Age $>$ 65No. (\%) & $14(35)$ \\
\hline Sex No.(\%) & $12(30)$ \\
Female & $28(70)$ \\
Male & \\
\hline Smoke-No.(\%) & $8(20)$ \\
No & $32(80)$ \\
Yes & \\
\hline Alcohol-No.(\%) & $36(90)$ \\
No & $4(10)$ \\
Yes & \\
\hline WHO-Performance state- No.(\%) & $18(45)$ \\
0 & $12(30)$ \\
1 & $10(25)$ \\
2 & \\
\hline Sites of metastasis- No.(\%) & $12(30)$ \\
Peritoneal with ascites & $16(40)$ \\
Liver & $8(20)$ \\
Lung & $3(7.5)$ \\
Bone & $1(2.5)$ \\
Distant LN (supraclavicualr) & $27(67.5)$ \\
\hline No. of metastatic sites- No.(\%) & $13(32.5)$ \\
Solitary & $5(12.5)$ \\
Multiple & $12(30)$ \\
\hline Presenting symptoms-No.(\%) & $34(85)$ \\
Jaundice & $30(75)$ \\
Ascites & \\
Pain & \\
Weight loss & \\
\hline Intervention needed-No. (\%) & \\
Billiary stent & \\
Tapping ascetic fluid & \\
\hline & \\
\hline
\end{tabular}

Between January 2014 and January 2018, 40 patients were enrolled in our clinic (NCC) and received the XELIRINOX treatment with the median follow-up was 15 months (range 2-48 months). The baseline characteristics are listed in Table 1 . The range of age was $40-75$, with a mean age of 61.3 years old .Twenty-six (65\%) patients were 65 years old and below, but $14(35 \%)$ were 66 years age or older. Twelve (30\%) patients were female, while 28
(70\%) were male. Thirty two (80\%) patients were smoky, while $8(20 \%)$ were nonsmoker. Thirty six $(90 \%)$ patients were non-alcoholic, while only $4(10 \%)$ were drink alcohol. The majority of the patients $18(45 \%)$ demonstrated a WHO-PS of 0 while $12(30 \%)$ had WHO-PS of 1 and remaining $10(25 \%)$ had WHO-PS of 2 . Two-third had a solitary metastatic site while remaining $13(32.5 \%)$ had multiple metastatic sites. The most common metastatic site was the liver, which diagnosed in $16(40 \%)$ patients while bone \& distant LN found with lowest percentage 3(7.5\%) \& $1(2.5 \%)$ respectively, and $12(30 \%)$ had peritoneal metastasis with malignant ascites and remaining $8(20 \%)$ had lung metastasis. Twenty-eight (70\%) patients presented with abdominal pain when diagnosed, and five patients $(12.5 \%)$ placed biliary stent or catheter because of jaundice and $12(30 \%)$ underwent tapping of ascetic fluid before chemotherapy start.

Baseline tumor marker results, including CA19-9, CEA, are also listed in Table 2. Thirty-six - patients (90\%) were found with elevated CA19-9 levels prior to start CT, but only four patients $(10 \%)$ had normal CA19-9. On the other hand, only eleven patients $(27.5 \%)$ found with elevated CEA, but remaining twenty-nine patients $(72.5 \%)$ had normal baseline CEA. The detailed patient characteristics are listed in Table 2.

Table 2. Objective Response rate, No. $=36$

\begin{tabular}{|l|c|}
\hline \multicolumn{1}{|c|}{ Response } & No. of patients (\%) \\
\hline CR & $1(2.5 \%)$ \\
\hline PR & $13(32.5 \%)$ \\
\hline SD & $12(30 \%)$ \\
\hline PD & $14(35 \%)$ \\
\hline ORR & $14(35 \%)$ \\
\hline CBR & $26(65 \%)$ \\
\hline Median PFS in months (CI) & $7.5(7.05-7.94)$ \\
\hline Median OS in months(CI) & $10.5(9.19-11.80)$ \\
\hline
\end{tabular}

\subsection{Treatment Delivery}

The median overall treatment period was 5.5 months and ranged from 1-6 months. The number of CT cycles ranged from 2-12 cycles with a median of 11 cycles. Treatment delay was present in $40(100 \%)$ patients. The duration of treatment delay ranged from 1 to 10 weeks with a median of 5 weeks.

All $40(100 \%)$ patients needed hospitalization due to toxicity during the treatment course .The duration of hospitalization ranged from 3 to 14 days with a median of 9 days. It looks interesting that thirty-six (90\%) patients can tolerate and completed the full course of 12 cycles CT regimen ,but unfortunately four (10\%) of patients received only up to four cycles.

\subsection{Efficacy}

Four patients $(10 \%)$ were dying in the early treatment 
period within the first two months with four cycles chemotherapy and cannot restaged. Thirty-six patients (90\%) were restaged after XELIRINOX treatment. The response of these patients was evaluated and is shown in Table 2. We found CR was observed, only in one patient out of $40(2.5 \%)$ and $13(32.5 \%)$ patients achieved a PR. Twelve $(30 \%)$ patients persisted stable disease. Unfortunately, 14 out of $40(35 \%)$ had progressive disease.

Objective response rate (ORR) was (35\%) and clinical benefit rate (CBR) was (65\%). The response to therapy is summarized in Table 2. Ten patients had to be taken off treatment due to disease progression and best supportive care was administered to him, then later on died. Four $(10 \%)$ patients were early dead, so the final total number of deaths were 14 (35\%) patients.

\subsection{Toxicity}

In general, the incidence of adverse events was $100 \%$ (No.= 40). Regarding hematological toxicity, anemia was the most frequent one, with an incidence of $60 \%$. Followed by thrombocytopenia occur in $40 \%$ of patients, but both of them of grade 1 . For neutropenia, we observed 12 patients (30\%) with grade $3 / 4$ toxicities, but neutropenic fever occurs in only four $(10 \%)$ patients. On the other hand, the most frequent non-hematological toxicity was nausea occurs in 26 patients $(65 \%)$ with grade $1 / 2$, also vomiting occurs in 18 patients $(45 \%)$ with grade $1 / 2$, with very low percentage of grade $3 / 4$ nausea\& vomiting occurs in two patients $(5 \%)$ probably due to the combination therapy of ondansetron, dexamethasone during the $\mathrm{CT}$ and oral fluorouracil content in this regimen. Diarrhea occurs in 18 patients $(65 \%)$ with grade $1 / 2$ and occurs in 12 patients (30\%) with grade $3 / 4$. Other adverse events occur more frequently in most patients, but of grade $1 / 2$ as, fatigue, anorexia, poor appetite \& Stamatis. The incidence of weight loss $\geq 5 \%$ occur in half of our patients. The incidence of sensory neuropathy was high and occurs in 30 patients $(75 \%)$ but of grade 1/2. Lastly hand and foot syndrome occurs in only 6 patients $(15 \%)$ of grade $1 / 2$ with no patients with grade 3/4. Totally, 12 patients $(30 \%)$ received G-CSF treatment before or after chemotherapy.

Table 3. Adverse events during treatment, No. $=40$

\begin{tabular}{|c|c|c|c|c|}
\hline Adverse events & \multirow{2}{*}{ Grade 1} & \multirow{2}{*}{ Grade 2} & \multirow{2}{*}{ Grade 3} & \multirow{2}{*}{ Grade 4} \\
\hline Hematological toxicityNo.-(\%) & & & & \\
\hline Anemia & $24(60 \%)$ & $8(20 \%)$ & $2(5 \%)$ & 0 \\
\hline Neutropenia & $4(10 \%)$ & $12(30 \%)$ & $6(15 \%)$ & $6(15 \%)$ \\
\hline Thrombocytopenia & $16(40 \%)$ & $2(5 \%)$ & $2(5 \%)$ & 0 \\
\hline Neutropenic fever & \multicolumn{4}{|c|}{$4(10 \%)$} \\
\hline \multicolumn{5}{|l|}{ Non Hematological toxicity No.-(\%) } \\
\hline Nausea & $20(50 \%)$ & $6(15 \%)$ & $2(5 \%)$ & 0 \\
\hline Vomiting & $12(30 \%)$ & $6(15 \%)$ & $2(5 \%)$ & 0 \\
\hline Stomitis & $8(20 \%)$ & $8(20 \%)$ & 0 & 0 \\
\hline Diarrhea & $14(35 \%)$ & $12(30 \%)$ & $8(20 \%)$ & $4(10 \%)$ \\
\hline Poor appetite & $12(30 \%)$ & $8(20 \%)$ & 0 & 0 \\
\hline Anorexia & $8(20 \%)$ & $12(30 \%)$ & $2(5 \%)$ & 0 \\
\hline Weight loss $\geq 5 \%$ & $20(50 \%)$ & & & \\
\hline Sensory Neuropathy & $20(50 \%)$ & $10(25 \%)$ & $2(5 \%)$ & 0 \\
\hline Fatigue & $8(20 \%)$ & $14(35 \%)$ & $2(5 \%)$ & 0 \\
\hline Hand and Foot Syndrome & $4(10 \%)$ & $2(5 \%)$ & 0 & 0 \\
\hline
\end{tabular}




\subsection{Survival}

Median follow-up was 15 months (range 2-48). 4(10\%) patients died in the early treatment period and completed up to four chemotherapy cycles and can't restage. Thirty six (90\%) patients continue and completed all CT cycles. The median PFS was 7.5 months (95\% CI: 7.059- 7.941), figure (1). Regarding OS, median OS was 10.5 months (95\% CI: 9.193-11.807), figure (2)

\section{Survival Function}

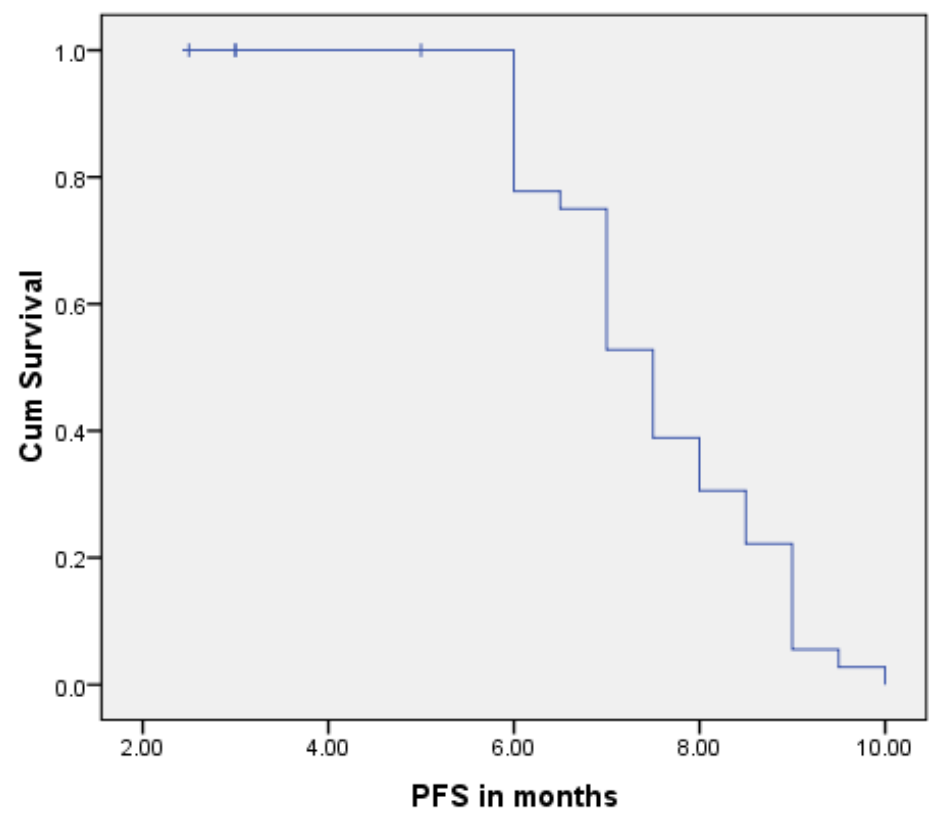

$\neg$ Survival Function + Censored

Figure 1. Kaplan-Meier curve of Progression free survival

Survival Function

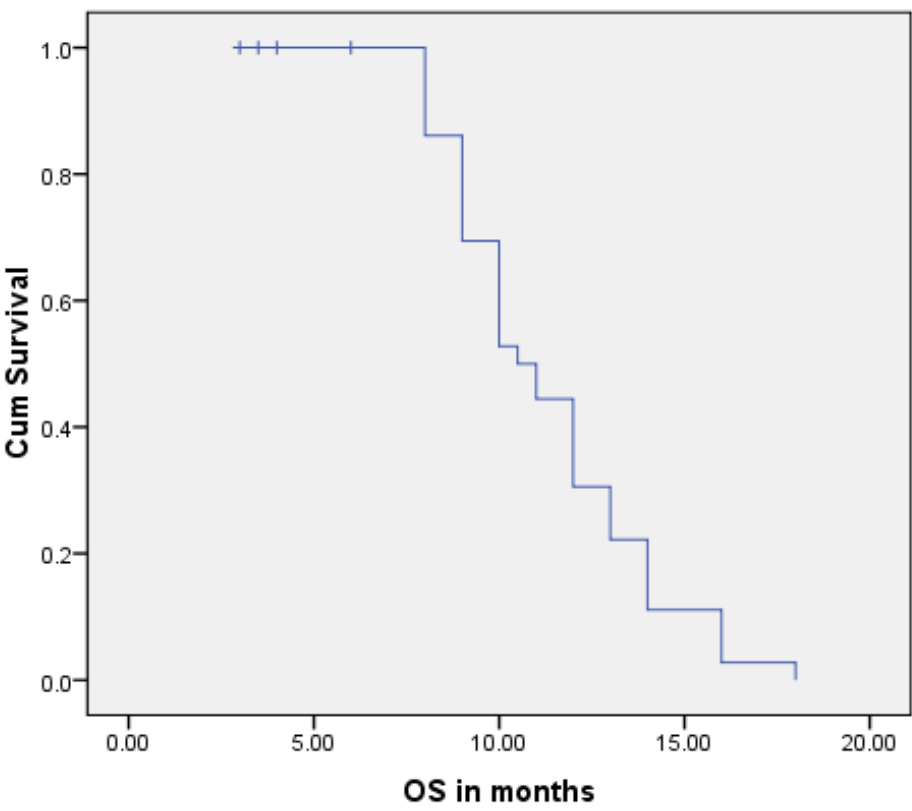

$\neg$ Survival Function + Censored

Figure 2. Kaplan-Meier curve of Overall survival. 


\subsection{Correlation}

There is none significant correlation between PFS and age, $(r=0.028)$, and $\mathrm{P}=0.866$ as showing in the table (4). There is significant negative correlation between PFS and WHO-PS, $(r=-0.365)$ and $P=0.020$. There is significantly negative correlation between PFS and number of metastatic sites, $(r=-0.309)$ and $\mathrm{P}=0.050$. There is highly significantly negative correlation between PFS and normal baseline tumor marker, $(\mathrm{r}=-0.471)$ and $\mathrm{P}=0.002$. Also there is highly significantly positive correlation between PFS and tumor marker reduction during treatment, $(r=$ $0.525)$ and $\mathrm{P}=0.001$. There is none significant correlation between PFS and weight loss $\geq 5 \%$ during treatment, $(r=$ 0.212 ) and $P=0.188$. Finally, there is highly significantly strong positive correlation between PFS and any treatment response $(\mathrm{r}=0.507)$ and $\mathrm{P}=0.001$.

Table 4. Correlation of PFS and OS to patient's characteristics

\begin{tabular}{|l|c|c|c|c|}
\hline & \multicolumn{2}{|c|}{ PFS in months } & \multicolumn{2}{c|}{ OS in months } \\
\hline Characteristic & $\boldsymbol{r}$ & $\boldsymbol{P}$ & $\boldsymbol{r}$ & $\boldsymbol{P}$ \\
\hline Age in years & 0.028 & $\begin{array}{c}0.866 \\
\mathrm{NS}\end{array}$ & 0.431 & $\begin{array}{c}0.005 \\
\text { HS }\end{array}$ \\
\hline WHO-Performance State & -0.365 & $\begin{array}{c}0.020 \\
\mathrm{~S}\end{array}$ & -0.536 & $\begin{array}{c}<0.001 \\
\text { HS }\end{array}$ \\
\hline Number of metastatic sites & -0.309 & $\begin{array}{c}0.050 \\
\mathrm{~S}\end{array}$ & -0.535 & $\begin{array}{c}<0.001 \\
\text { HS }\end{array}$ \\
\hline $\begin{array}{l}\text { Normal baseline tumor } \\
\text { Marker }\end{array}$ & -0.471 & $\begin{array}{c}0.002 \\
\mathrm{HS}\end{array}$ & 0.261 & $\begin{array}{c}0.103 \\
\mathrm{NS}\end{array}$ \\
\hline Decrease tumor marker & 0.525 & $\begin{array}{c}0.001 \\
\mathrm{HS}\end{array}$ & 0.724 & $\begin{array}{c}<0.001 \\
\mathrm{HS}\end{array}$ \\
\hline $\begin{array}{l}\text { Weight loss }>5 \% \text { during } \\
\text { treatment }\end{array}$ & 0.212 & $\begin{array}{c}0.188 \\
\mathrm{NS}\end{array}$ & 0.769 & $\begin{array}{c}<0.001 \\
\mathrm{HS}\end{array}$ \\
\hline Clinical benefit rate & 0.507 & $\begin{array}{c}0.001 \\
\mathrm{HS}\end{array}$ & 0.796 & $\begin{array}{c}<0.001 \\
\text { HS }\end{array}$ \\
\hline
\end{tabular}

$r$ : Spearman correlation coefficient; NS: not significant at $P \leq 0.05$; S: significant at $P \leq 0.05$; HS: highly significant at $P \leq 0.01$

On the other hand, regarding OS correlation, there is strong positive correlation between OS and age, $(\mathrm{r}=$ 0.431 ), and $\mathrm{P}=0.005$ as showing in the Table 4 . There is highly significant negative correlation between OS and WHO-PS, $(r=-0.536)$ and $\mathrm{P}=<0.001$. There is highly significantly negative correlation between OS and number of metastatic sites, $(\mathrm{r}=-0.535)$ and $\mathrm{P}=<0.001$. There is none significantly correlation between OS and normal baseline tumor marker, $(r=0.261)$ and $P=0.103$. Also there is highly significantly positive correlation between $\mathrm{OS}$ and tumor marker reduction during treatment, $(\mathrm{r}=$ $0.724)$ and $\mathrm{P}=<0.001$. There is highly significant positive correlation between OS and weight loss $\geq 5 \%$ during treatment, $(r=0.769)$ and $\mathrm{P}=<0.001$. Finally, there is highly significantly strong negative correlation between OS and any treatment response $(\mathrm{r}=0.796)$ and $\mathrm{P}=<0.001$.

\section{Discussion}

Metastatic PAC patients have extremely short survival, and few treatment modalities have been established in such clinical scenario. FOLFIRINOX chemotherapy can produce remarkable efficacy in metastatic PAC patients with significantly increased survival, compared to gemcitabine monotherapy and is a promising strategy for these unfortunates. However, the application of FOLFIRINOX is largely limited by its considerable toxicity, as well as $5-\mathrm{FU}$ had to be administered as a $48-\mathrm{h}$ continuous infusion by a central venous catheter to make the combination feasible as noticed by Conroy et al. [10]

As a consequent, many oncologists are hesitating to prescribe FOLFIRINOX for metastatic PAC patients, although it may have better efficacy to combat tumor therefore very limited metastatic PAC patients have tried the FOLFIRINOX regimen in some institutions.

To make FOLFIRINOX more practicable, many investigators have made their efforts to enhance patients' tolerance to this $\mathrm{CT}$ through various ways as stated by Gunturn et al. [24]. Mahaseth et al. significantly reduced the incidence of grade 3/4 neutropenia and diarrhea without compromising the efficiency of FOLFIRINOX simply by removing fluorouracil bolus and using G-CSF at the same time [15]. Stein et al. reduced the doses of both irinotecan and 5 -FU bolus by $25 \%$, and found that the incidences of neutropenia, vomiting and fatigue were significantly decreased [25]. Other modification strategy for advanced PC includes avoiding the use of irinotecan and it may be referenced in metastatic PAC patients as mentioned by El-Hadaad et al. [26]

Our study is the first trial to evaluating the activity of a first-line triplet combination of alternated XELIRINOXby giving same the oxaliplatin dose, but reducing irinotecan to $75 \%$ of the full dose and substituted capcitabine instead of 5-FU (that used in FOLFIRINOX) for the purpose of improving tolerability.

Meanwhile, our modification maintained considerable efficacy of XELIRINOX, with an ORR of 35\% and CBR of $65 \%$ \& prolonged survival with acceptable toxicity.

In the current study, we enrolled 40 metastatic PAC patients and provided a novel alternative strategy for FOLFIRINOX according to our experience. In our cohort, the median OS and PFS were 10.5 and 7.5 months, respectively, comparable to the previous studies.

Alternated XELIRINOX is also supposed to reduce adverse events. Conroy et al. reported that grade 3/4 adverse events with FOLFIRINOX treatment are neutropenia (45.7\%), fatigue (17.8\%), vomiting (14.5\%), and diarrhea (12.7\%) [27]. We showed that only $30 \%$ of patients receiving XELIRINOX suffered grade 3/4 neutropenia, which was usually short lasting and rarely complicated, and only $5 \%$ grade 3 fatigue without grade 4 , vomiting occurs in 50\% grade 1-3 without grade 4 . Furthermore sensory neuropathy occurs much lower in our study with only (5\%) grade 3 without grade 4 compared to (9\%) occur grade 3-4 in FOLFIRINOX. But the incidence of hand and foot syndrome occurs in (15\%) of our patients, which only of grade 1-2.

The major concern with the XELIRINOX regimen is the gastrointestinal toxicity, in particular in terms of grade 3/4 
$(30 \%)$ was observed more frequently and higher than FOLFIRNOX, but it is manageable and acceptable. We believe that the lowering Irinotecan dose to $75 \%$ and use one week capcitabine in addition to the use of G-CSF contributed to the reduction of grade $3 / 4$ neutropenia and diarrhea.

The relatively low risk of grade $3 / 4(5 \%)$ hematological adverse events (anemia, thrombocytopenia and neutropenic fever) and none CT-related treatment cessation or death suggested a good tolerability of our XELIRINOX regimen.

\section{Summary}

We showed that the substitution of capecitabine for infusional 5-FU, in combination with lowering irinotecan dose to $75 \%$ and constant oxaliplatin dose, retained an interesting activity in the first-line treatment of metastatic PACand well tolerated \& could replace the need for an implanted central venous catheter with an ORR of $35 \%$, median OS of 10.5 months, and PFS of 7.5 months. However, the incidence of grade 3-4 diarrhoea experienced with the XELIRINOXregimen seems higher than that with FOLFIRINOX, but it was manageable and acceptable in addition to that, the regimen with the oral fluoropyrimidine seemed less manageable than that with infusional 5-FU. Therefore, a triple-drug combination of CPT-11 and L-OHP with capecitabine instead of infusional 5-FU as we used is a preferable alternative to FOLFIRINOX, and can be considered for patients with metastatic PAC. The European Organization for Research and Treatment of Cancer (EORTC) QLQ-C30 questionnaire may be needed to obtain a better evaluation of the quality of life of patients receiving XELIRINOX treatment. To our knowledge, this is the first prospective study aiming to evaluate the efficacy of XELIRINOX in metastatic PAC patients. More studies involving well-designed randomized controlled trials are required to validate these results and to display whether XELIRINOX regimen used as an alternative to FOLFIRINOX in advanced PAC.

\section{Abbreviations}

BSC: best supportive care

CT: Chemotherapy

G-CSF: Colony stimulating factor

GI: Gastrointestinal

IV: Intravenous

PAC: Pancreatic adenocarcinoma

PC: Pancreatic cancer

RECIST: Response evaluation criteria in solid tumors WHO-PS: World Health Organization-Performance state.

\section{Acknowledgements}

I would like to thank my Colleague Assistant professor Thaer Wally for his assistance with information collection and statistical calculations also thanks for other sub-staff for considerable aid and patient support and finally thanks for our patients accepted to treatment and remained in close follow up.

\section{Conflict of Interest}

There is no any potential conflict of interest.

\section{Ethical Clearance}

Taken from our scientific university committee (Jaber Bin Hayyan Medical University)

\section{REFERENCES}

[1] Islami F, Goding Sauer A, Miller KD, Siegel RL, Fedewa SA, Jacobs EJ, McCullough ML, Patel AV, Ma J, Soerjomataram I, Flanders WD. Proportion and number of cancer cases and deaths attributable to potentially modifiable risk factors in the United States. CA: a cancer journal for clinicians. 2018 Jan; 68(1): 31-54.

[2] Siegel RL, Miller KD, Jemal A. Cancer statistics, 2017. CA: a cancer journal for clinicians. 2017 Jan 1; 67(1): 7-30.

[3] Simard EP, Ward EM, Siegel R, Jemal A. Cancers with increasing incidence trends in the United States: 1999 through 2008. CA: a cancer journal for clinicians. 2012 Mar $1 ; 62(2): 118-28$.

[4] Eheman C1, Henley SJ, Ballard-Barbash R, Jacobs EJ, Schymura MJ, Noone AM, Pan L, Anderson RN, Fulton JE, Kohler BA, Jemal A, Ward E, Plescia M, Ries LA, Edwards BK. Annual Report to the Nation on the status of cancer, 1975-2008, featuring cancers associated with excess weight and lack of sufficient physical activity . Cancer 2012; 118: 2338-2366.

[5] Worni M, Guller U, White RR, Castleberry AW, Pietrobon R, Cerny T, Gloor B, Koeberle D. Modest improvement in overall survival for patients with metastatic pancreatic cancer: a trend analysis using the surveillance, epidemiology, and end results registry from 1988 to 2008 . Pancreas. 2013 Oct 1; 42(7): 1157-63.

[6] Fogel EL, Shahda S, Sandrasegaran K, DeWitt J, Easler JJ, Agarwal DM, Eagleson M, Zyromski NJ, House MG, Ellsworth S, El Hajj I, O’Neil BH, Nakeeb A, Sherman S. A multidisciplinary approach to pancreas cancer in 2016: a review. The American journal of gastroenterology. 2017 Apr; 112(4): 537.

[7] Warsame R, Grothey A. Treatment options for advanced pancreatic cancer: a review. Expert review of anticancer therapy. 2012 Oct 1; 12(10):1327-36. 
[8] Burris HA,Moore MJ, Andersen J, Green MR, Rothenberg ML, Modiano MR, Cripps MC, PortenoyRK, StornioloAM, TarassoffP, Nelson R, Stephens AD, Von HoffDD. Improvements in survival and clinical benefit with gemcitabine as first- line therapy for patients with advanced pancreas cancer: A randomized trial. Journal of Clinical Oncology 15, 2403-2413 (1997).

[9] Di Marco M, Di Cicilia R, Macchini M, Nobili E, Vecchiarelli S, Brandi G, Biasco G. Metastatic pancreatic cancer: is gemcitabine still the best standard treatment? Oncology reports. 2010 May 1; 23(5):1183-92.

[10] Conroy T, Desseigne F, Ychou M, Bouché O, Guimbaud R, Bécouarn Y, Adenis A, Raoul JL, Gourgou-Bourgade S, de la Fouchardière $\mathrm{C}$, Bennouna J, Bachet JB, Khemissa-Akouz F, Péré-Vergé D, Delbaldo C, Assenat E, Chauffert B,Michel P, Montoto-Grillot C, Ducreux M. FOLFIRINOX versus gemcitabine for metastatic pancreatic cancer. New England Journal of Medicine. 2011 May 12; 364(19): 1817-25.

[11] Von Hoff DD, Ervin T, Arena FP, Chiorean EG, Infante J, Moore M, Seay T, Tjulandin SA, Ma WW, Saleh MN, Harris M, Reni M, Dowden S, Laheru D, Bahary N, Ramanathan RK, Tabernero J, Hidalgo M, Goldstein D, Van Cutsem E, et al. Increased survival in pancreatic cancer with nab-paclitaxel plus gemcitabine. New England Journal of Medicine. 2013 Oct 31; 369(18):1691-703.

[12] Chan K, Shah K, Lien K, Coyle D, Lam H, Ko YJ. A Bayesian meta-analysis of multiple treatment comparisons of systemic regimens for advanced pancreatic cancer. PloS one. 2014 Oct 6; 9(10):e108749.

[13] Gresham GK, Wells GA, Gill S, Cameron C, Jonker DJ. Chemotherapy regimens for advanced pancreatic cancer: a systematic review and network meta-analysis. BMC cancer. 2014 Dec; 14(1):471.

[14] Conroy T, Gavoille C, Samalin E, Ychou M, Ducreux M. The role of the FOLFIRINOX regimen for advanced pancreatic cancer. Current oncology reports. 2013 Apr 1; 15(2):182-9.

[15] Mahaseth H, Brutcher E, Kauh J, Hawk N, Kim S, Chen Z, Kooby DA, Maithel SK, Landry J, El-Rayes BF. Modified FOLFIRINOX regimen with improved safety and maintained efficacy in pancreatic adenocarcinoma. Pancreas. 2013 Nov 1; 42(8): 1311-5.

[16] Stein SM, James ES, Deng Y, Cong X, Kortmansky JS, Li J, Staugaard C, Indukala D, Boustani AM, Patel V, Cha CH, Salem RR, Chang B, Hochster HS, Lacy J. Final analysis of a phase II study of modified FOLFIRINOX in locally advanced and metastatic pancreatic cancer. British journal of cancer. 2016 Mar; 114(7): 809.

[17] Mazard T, Ychou M, Thezenas S, Poujol S, Pinguet F, Thirion A, Bleuse JP, Portales F, Samalin E, Assenat E. Feasibility of biweekly combination chemotherapy with capecitabine, irinotecan, and oxaliplatin in patients with metastatic solid tumors: results of a two-step phase I trial: XELIRI and XELIRINOX. Cancer chemotherapy and pharmacology. 2012 Mar 1; 69(3): 807-14.

[18] Cassidy J, Twelves C, Cutsem EV, Hoff P, Bajetta E, Boyer M, Bugat R, Burger U, Garin A, Graeven U, McKendrick J, MarounJ, MarshallJ, Osterwalder B, Pérez-MangaG, RossoR, Rougier P, Schilsky RL.Gastrointestinal tumors-First-line oral capecitabine therapy in metastatic colorectal cancer: A favorable safety profile compared with intravenous 5-fluorouracil/leucovorin. Annals of Oncology-English Edition. 2002; 13(4): 566-75.

[19] Koopman M, Antonini NF, Douma J, Wals J, Honkoop AH, Erdkamp FL, de Jong RS, Rodenburg CJ, Vreugdenhil G, Loosveld OJ, van Bochove A, Sinnige HA, Creemers GJ, Tesselaar ME, Slee PH, Werter MJ, Mol L, Dalesio O, Punt CJ (2007) Sequential vs combination chemotherapy with capecitabine, irinotecan and oxaliplatin in advanced colorectal cancer (CAIRO): a phase III randomised controlled trial. Lancet 370: 135-142

[20] Mackean M, Planting A, Twelves C, Schellens J, Allman D, Osterwalder B, Reigner B, Griffin T, Kaye S, Verweij J. Phase I and pharmacologic study of intermittent twice-daily oral therapy with capecitabine in patients with advanced and/or metastatic cancer. Journal of Clinical Oncology. 1998 Sep; 16(9): 2977-85.

[21] Traina TA, Theodoulou M, Feigin K, Patil S, Tan KL, Edwards C, Dugan U, Norton L, Hudis C. Phase I study of a novel capecitabine schedule based on the Norton-Simon mathematical model in patients with metastatic breast cancer. Journal of Clinical Oncology. 2008 Apr 10; 26(11): 1797-802.

[22] Scheithauer W, Kornek GV, Raderer M, Schüll B, Schmid K, Kovats E, Schneeweiss B, Lang F, Lenauer A, Depisch D. Randomized multicenter phase II trial of two different schedules of capecitabine plus oxaliplatin as first-line treatment in advanced colorectal cancer. Journal of clinical oncology. 2003 Apr 1; 21(7): 1307-12.

[23] Eisenhauer EA, Therasse P, Bogaerts J, Schwartz LH, Sargent D, Ford R, Dancey J, Arbuck S, Gwyther S, Mooney M, Rubinstein L, Shankar L, Dodd L, Kaplan R, Lacombe D, Verweij J . New response evaluation criteria in solid tumours: revised RECIST guideline (version 1.1). European journal of cancer. 2009 Jan 1; 45(2): 228-47.

[24] Gunturu KS, Yao X, Cong X, Thumar JR, Hochster HS, Stein SM, Lacy J. FOLFIRINOX for locally advanced and metastatic pancreatic cancer: single institution retrospective review of efficacy and toxicity. Medical oncology. 2013 Mar 1; 30(1): 361 .

[25] Stein SM, James ES, Deng Y, Cong X, Kortmansky JS, Li J, Staugaard C, Indukala D, Boustani AM, Patel V, Cha CH, Salem RR, Chang B, Hochster HS, Lacy J. Final analysis of a phase II study of modified FOLFIRINOX in locally advanced and metastatic pancreatic cancer. British journal of cancer. 2016 Mar; 114(7): 809.

[26] El-Hadaad HA, Wahba HA. Oxaliplatin plus 5-fluorouracil and folinic acid (OFF) in gemcitabine-pretreated advanced pancreatic cancer: a phase II study. Journal of gastrointestinal cancer. 2013 Sep 1; 44(3): 313-7.

[27] Suker M, Beumer BR, Sadot E, Marthey L, Faris JE, Mellon EA, El-Rayes BF, Wang-Gillam A, Lacy J, Hosein PJ, Moorcraft SY, Conroy T, Hohla F, Allen P, Taieb J, Hong TS, Shridhar R, Chau I, van Eijck CH, Koerkamp BG. FOLFIRINOX for locally advanced pancreatic cancer: a systematic review and patient-level meta-analysis. The Lancet Oncology. 2016 Jun 1; 17(6): 801-10. 